\title{
Kein Hinweis auf kumulative kognitive Defizite
}

Fragestellung: Nehmen kognitive Defizite bei steigender Anzahl von Elektrokonvulsionstherapie (EKT)-Behandlungen zu?

Hintergrund: Die EKT ist die akut wirksamste Behandlung bei schwerer Depression mit Remissionsraten von etwa $50 \%$. Allerdings wird die EKT in den Medien häufig als eine barbarische Behandlung dargestellt und deren kognitive Nebenwirkungen als tiefgreifend und stark behindernd berichtet. Mit Ausnahme der retrograden Amnesie sind jedoch alle anderen kognitiven Defizite durch die EKT passager und bilden sich meist innerhalb von drei Tagen wieder vollständig zurück. Es ist bisher wenig über mögliche kumulative kognitive Defizite durch länger angewendete EKT (mehr als zwölf Behandlungen) oder Erhaltungs-EKT bekannt.

Patienten und Methodik: Diese prospektive, nicht verblindete, nicht kontrollierte, offene naturalistische Studie wurde über zehn Jahre an der Cardiff Universität durchgeführt. Neun unterschiedliche kognitive Tests wurden nach Empfehlung des ECT Accredation Service (ECTAS) des Royal College of Psychiatrists gemacht. Allen Patienten, bei denen die Indikation zur EKT gestellt wurde, ist diese kognitive Testung angeboten worden, die vor Beginn, eine Woche nach und drei Monate nach Ende der EKT durchgeführt wurde. Patienten mit ErhaltungsEKT wurden jährlich getestet. Patienten mit der Diagnose Schizophrenie und nicht fließend englisch sprechende Patienten wurden ausgeschlossen. Die Hamilton Rating Scale for

Kirov GG, Owen L, Ballard $\mathrm{H}$ et al. Evaluation of cumulative cognitive deficits from electroconvulsive therapy. Brit J Psychiatry 2016; 208: $266-70$
Depression (HRSD-24) wurde als klinische Skala zur Bewertung der Schwere der Depression verwendet. Zur statistischen Analyse wurde eine (lineare mixed-effect, li- neare gemischte) Regression mit vier Kovariaten gerechnet. Kovariaten als mögliche die Kognition beeinflussende Faktoren waren Alter, Anzahl der Tage seit der letzten EKT, Schwere der Depression und Anzahl der Testungen, die ein Patient insgesamt im Verlauf der Studie durchgeführt hat. Als sekundäre Analyse wurde die erste und letzte Testung eines Patienten mit einem („paired sample“, gepaarte Stichproben) t-Test verglichen.

Ergebnisse: Das Durchschnittsalter der Patienten zum Zeitpunkt der letzten Testung betrug 56,3 Jahre (SD 15,4, Range 21 - 88). 71,9\% der Patienten waren weiblich. 83,4\% der Patienten $(n=166)$ hatten eine unipolare und 33 Patienten eine bipolare Störung bei aktueller depressiver Episode. Die Patienten hatten 15,3 EKT (SD 23,2, Range 0-186) zum Zeitpunkt der Testung. 96 der 199 in die Analyse eingegangenen Patienten hatten mehr als zwölf EKT und elf mehr als 50 EKT.

Die Ergebnisse der einzelnen kognitiven Tests wurden in Bezug auf die Anzahl der EKT und die vier Kovariaten (Alter, Anzahl der Tage seit der letzten EKT, Schwere der Depression und Anzahl der Testungen, die ein Patient durchlaufen hat) ermittelt. Der Faktor mit dem größten Einfluss (acht von neun Tests) auf die Kognition war das Alter, gefolgt von der Schwere der Depression (sieben von neun Tests). Die Anzahl der vorherigen EKT hatte keinen signifikanten Effekt in den jeweiligen Tests. In allen Tests wurden Verbesserungen der Kognition gemessen. Diese waren am ehesten auf die Verbesserung der Schwere der Depression zurückzuführen (HRSD-24 von 29,1 vor EKT und 14,7 zum letzten Testzeitpunkt).

Schlussfolgerungen: Es besteht kein Anhalt für eine kumulative kognitive Verschlechterung mit zunehmender Anzahl von EKT-Behandlungen. Hingegen waren Alter und Schwere der Depression Faktoren, die die Ergebnisse der kognitiven Testung negativ beeinflusst haben.

\section{- Kommentar von Sarah Kayser, Mainz}

\section{Kognitive Langzeiteffekte bei EKT differenzierter hinterfragen}

Diese naturalistische Studie (Effectiveness-Studie) ist die erste, die über zehn Jahre kognitive Untersuchungen an Patienten durchführte, die wiederholt EKT erhielten. Damit ist ein quasiexperimentelles Design ohne Randomisierung mit der bisher größten Studienpopulation erfüllt. Durch dieses Design ist die Generalisierbarkeit der Ergebnisse gegeben. Jedoch sind kausale Zusammenhänge der Ergebnisse eingeschränkt zu treffen. Die Ergebnisse leisten einen entschiedenen Anteil dazu, um Ängsten vor bleibenden kognitiven Defiziten und der Stigmatisierung der EKT bei Patienten, Behandlern, Angehörigen und den Medien entgegenzuwirken. Einschränkend muss erwähnt werden, dass der Zusammenhang zwischen EKT und der kognitiven Störung, die am häufigsten Langzeitdefizite verursacht, die retrograde Amnesie, nicht untersucht wurde.

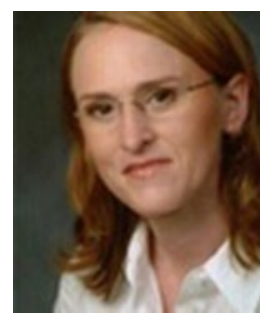

Dr. med. Sarah Kayser, MSc., Mainz

Klinik für Psychiatrie und Psychotherapie Universitätsmedizin Mainz E-Mail: sarah.kayser@unimedizin-mainz.de 